\title{
Simulation and Analysis of 75mm Gas-liquid Cyclone Flow Field
}

\author{
Liang Ma, Pen Qian, Jingping Wu, Zhaoyuan Bai, Qiang Yang. \\ State Environmental Protection Key Laboratory of Environmental Risk Assessment and Control on Chemical Process \\ East China University of Science and Technology \\ Shanghai, China \\ y.q.love@163.com, maliang3678@163.com
}

\begin{abstract}
The inside flow field of a gas-liquid cyclone separator with a diameter of $75 \mathrm{~mm}$ was simulated with the computational fluid dynamics (CFD). Results showed that a variation of inlet flow rate significantly changes the inside flow field structure and affects separation efficiency. The axial velocity of sections at four different positions was studied. All absolute values of the axial velocity increased with increased inlet flow rate. The regional line diagram of axial velocity shows that the increased inlet flow rate significantly increases the axial velocity area in the upward direction and decreases the same in the downward direction.
\end{abstract} velocity

Keywords- CFD; Gas-liquid cyclone; Simulation; Axial

\section{INTRODUCTION}

The gas-liquid cyclone separator is used to implement gas-liquid separation by centrifugal force with a simple structure and without moving parts. This equipment is easy to maintain and is high temperature and high pressure resistant [1, 2]. Therefore, it is widely used in various applications and industries, such as petrochemicals, coalfired power generation, and iron and steel metallurgy [3-5]. The inside of the cyclone separator is characterized by a 3D turbulent flow field divided into axial, radial, and tangential velocity. Axial velocity has a significant influence on the downward transmission and separation of particles in the cyclone separator; axial velocity can be divided into upward flow (upward direction) and downward flow (downward direction). The distribution of axial velocity is complicated. Most studies have reported that with an inverted V-shaped distribution, axial velocity at the separator wall is low and that at the center is the highest $[6,7]$.

With the development of computer simulation technology, an increasing number of scholars use computational fluid dynamics (CFD) to simulate the complicated 3D flow field inside the cyclone separator. Since Boysan [8] used an axis-symmetry and the algebraic stress model for predicting the field of the gas phase and fraction collection efficiency, a number of CFD simulations have been successfully applied by employing the different mathematic model to determine the features of flow field for cyclones [9-12].

In this article, CFD simulation technology was employed to simulate the flow field inside the gas-liquid separator with a diameter of $75 \mathrm{~mm}$ for the first time. Simulation with established models shows that the flow field and axial velocity inside the cyclone separator were changed. Similarly, the areas with different axial velocities changed under different processing volumes.

\section{MODEL}

A cylinder-on-cone cyclone with diameter of $75 \mathrm{~mm}$ was designed in this study. The cyclone geometry is shown in Figure 1. The detailed dimensions (units in $\mathrm{mm}$ ) of the cyclone are listed in Table 1.

The multiphase flow in a cyclone is quite complicated. In this work, velocity fields of the cyclone with different feeding flow rate were numerically simulated using FLUENT computational fluid dynamics (CFD) software. Some works of simulation of cyclone using the Reynolds stress model (RSM) have been reported to be suitable for modeling the flow in the cyclone in recent years [13-15]. Therefore, the Reynolds stress model (RSM) of the cyclone internal turbulent flow simulation is used in this paper. Gambit, which is the main preprocessor of Fluent, is used to create geometry, meshing and specifying boundary types of cyclone. The created geometry and meshing are shown in Figures 2 and 3. Boundary types are considered as inlet mass flow rate, overflow and underflow outlet pressure. The liquid concentration of feed aerosol was $5 \mathrm{~g} / \mathrm{m}^{3}$. Liquid droplet with different sizes varying between 1 and $10 \mu \mathrm{m}$ were injected from the feed inlet boundary zone along the surface. Using Fluent, the created geometry by Gambit can be read and simulation is done.
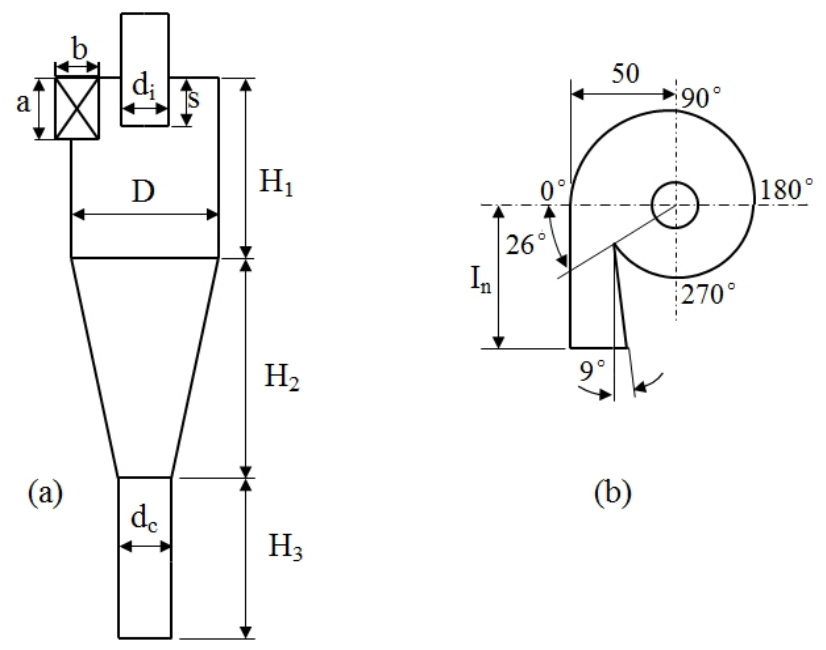

(b)

Figure 1. Cyclone structure (a) and the angular positions (b) 
TABLE I. DIMENSIONS OF THE CYCLONE

\begin{tabular}{cccccccccc}
\hline$D$ & $a$ & $b$ & $d_{i}$ & $d_{c}$ & $S$ & $H_{1}$ & $H_{2}$ & $H_{3}$ & $I_{n}$ \\
\hline 75 & 45 & 30 & 35 & 38 & 50 & 150 & 225 & 150 & 100 \\
\hline
\end{tabular}

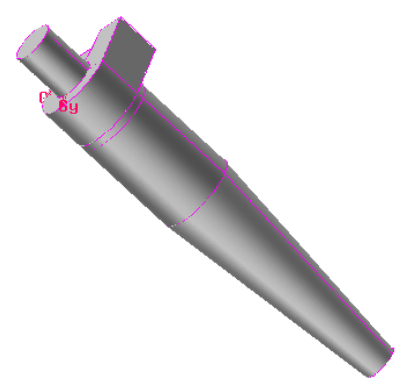

Figure 2. Cyclone geometry

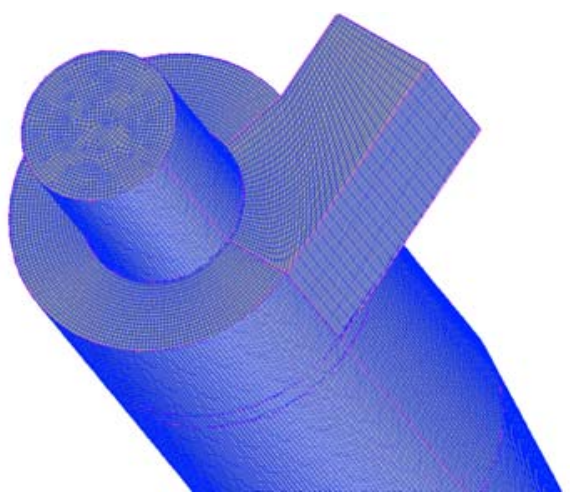

Figure 3. Cyclone mesh generation

\section{RESULTS AND DISCUSSION}

\section{A. Flow Pattern}

The patterns under different flows (Q) are shown in figure 4. As seen in the figure 4, the flow field turbulence inside the cyclone separator gradually increased with the addition of flow. Several vortexes inside the flow field were generated consequently. When the flow rate was $70 \mathrm{~m}^{3} / \mathrm{h}$,
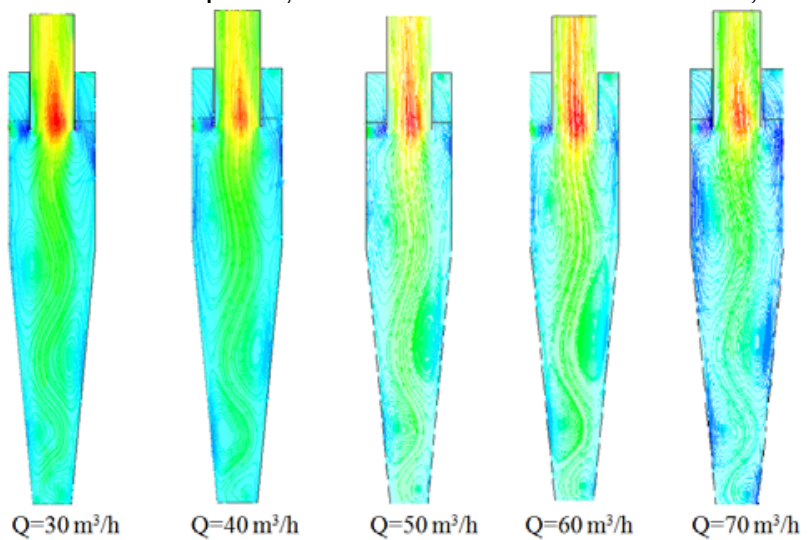

Figure 4. Flow pattern under different flows several vortexes with large coverage were generated. The swing amplitude at the end of the vortexes increased, thereby increasing the back-mixing and carriage of particles at the bottom of the cone and resulting in the decrease in separation efficiency of the cyclone separator. Thus, the cyclone separator is within the normal operation range with 30 $50 \mathrm{~m}^{3} / \mathrm{h}$.

\section{B. Distribution of Axial Velocity}

The position of vortex finder tip is defined as $Z=0$. The four- dimensional velocity distributions in the position $Z=-50$, $-100,-200$, and -300 of the cyclone is studied separately. The axial velocity simulation results are described as follows.
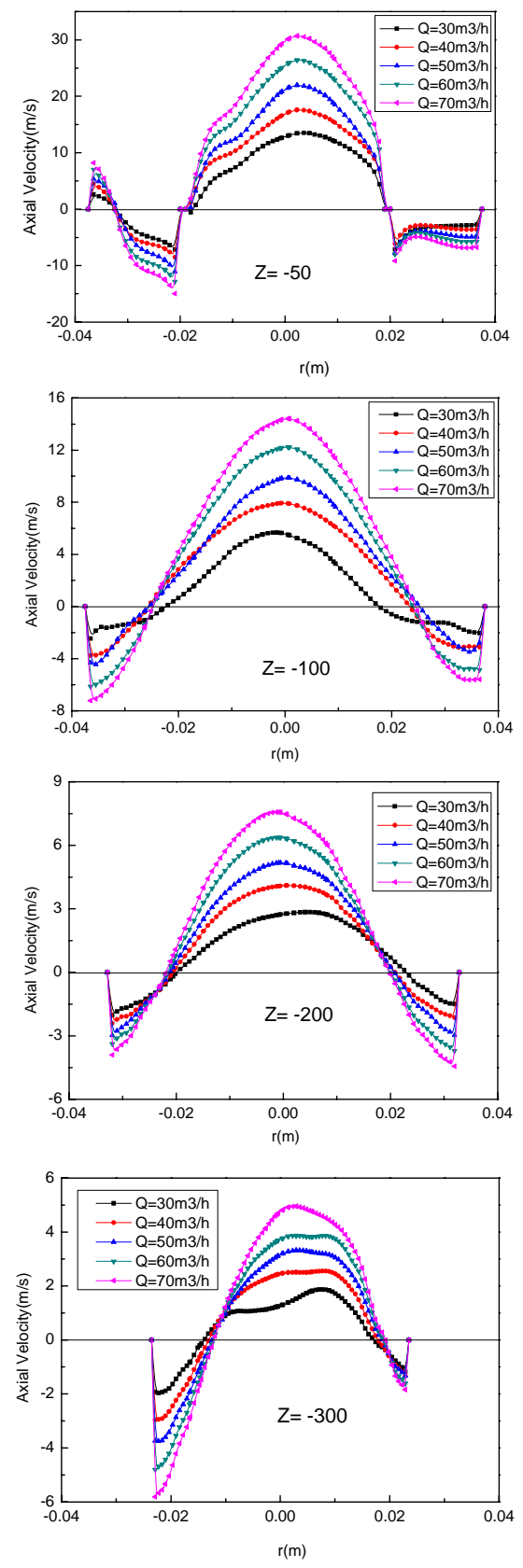

Figure 5. Distribution diagram of axial velocity in four section 
The axial velocity profiles in different cross-sections are shown in Figure 5. It can be seen that both the upward and downward velocity are increase with the inlet flow. The axial velocity in each cross-section basically shows a symmetric distribution, changing gradually from negative to positive in the direction from the wall to the center. The fluid along the wall moves to the underflow outlet, and the downward velocity increases with its radial distance from the cyclone axis. The fluid around the axis moves to the overflow outlet, and the upward velocity increases with its radial distance from the cyclone wall.

The difference of the $Z=-50$ section from the other three sections is attributed to the two regions with positive axial velocity found in the axial velocity distribution chart. That is, a smaller region with positive axial velocity exists in the $\mathrm{r}=-0.035$ region. The $\mathrm{Z}=-50$ and $\mathrm{r}=-0.035$ regions are just below the inlet of the cyclone separator. When the inlet flow is increased, the upward axial velocity is also improved.

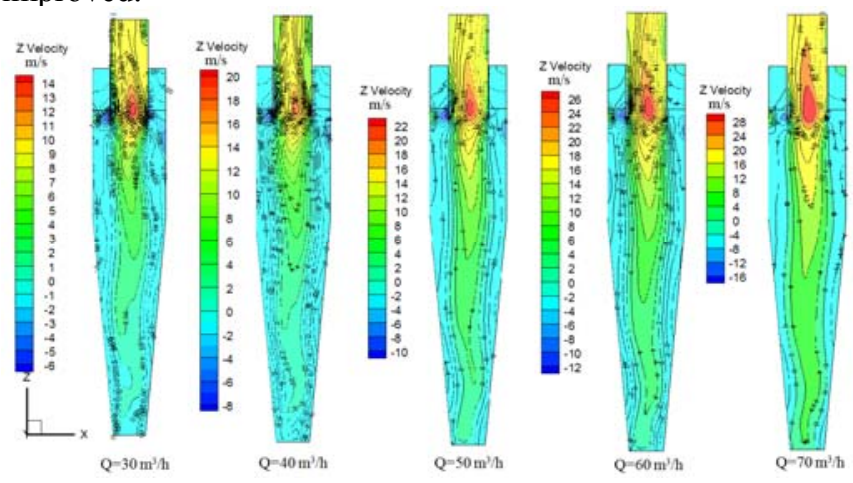

Figure 6. Regional line diagram of axial velocity

Fig. 6 shows that the increase in processing volume not only caused an apparent increase in absolute upward axial velocity, but also an evident increase in its coverage. The increase in processing volume also caused the absolute downward velocity to increase (from $6 \mathrm{~m} / \mathrm{s}$ to $16 \mathrm{~m} / \mathrm{s}$ ); however, its coverage decreased. When the flow rate was 60 and $70 \mathrm{~m}^{3} / \mathrm{h}$, most parts of the exit area at the bottom were covered by upward axial velocity, thereby causing the droplet particles to be back mixed to the exit at the top. Consequently, separation efficiency decreased. When the flow rate was $30 \mathrm{~m}^{3} / \mathrm{h}$, the exit area at the bottom was almost covered by downward axial velocity. This condition indicates that a large amount of gas flowed from the bottom. This amount is wasted raw gas material. Thus, the reasonable flow rate for the cyclone separator is $40-50 \mathrm{~m}^{3} / \mathrm{h}$.

\section{Grade efficiency}

The grade efficiency curves of cyclone are shown in Fig.7. It can be seen that the variation in inlet flow has no significant effect on the trend of the grade efficiency curve, and the grade efficiency is $100 \%$ when the particle size bigger than $10 \mu \mathrm{m}$. The sharpness of grade efficiency curve of $50 \mathrm{~m}^{3} / \mathrm{h}$ is slightly higher than other. The grade efficiency at flow rates of 30 and $40 \mathrm{~m}^{3} / \mathrm{h}$ is lower than that at flow rates of 50,60 , and $70 \mathrm{~m}^{3} / \mathrm{h}$. And the grade efficiency shows a slight decreasing trend when the flow rate exceeds $50 \mathrm{~m}^{3} / \mathrm{h}$. The cut size changes from $1.7 \mu \mathrm{m}$ to $2.8 \mu \mathrm{m}$ at different inlet flow. That is, the inlet flow has significantly effect on the cut size also.

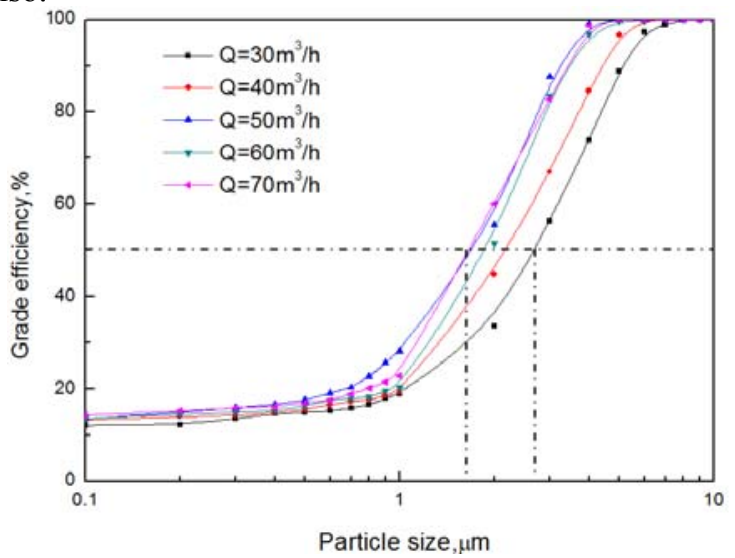

Figure 7. Grade efficiency curves of cyclone

\section{CONCLUSION}

The increase in flow rate caused the flow field turbulence inside the separator with a diameter of $75 \mathrm{~mm}$ to increase and generate a large vortex, which increased the back-mixing and carriage of particles at the bottom of the cone and caused a decrease in the separation efficiency of the cyclone separator. Axial velocity increased with the increase in flow rate and exhibited good symmetry. The upward axial velocity coverage clearly increased with the increase in flow rate. However, the downward axial velocity coverage decreased with the increase in flow rate. In consideration of these results, we conclude that the reasonable flow rate for the cyclone separator is $40-50 \mathrm{~m}^{3} / \mathrm{h}$.

\section{REFERENCES}

[1] S. Movafaghian, J. A. Jaua-Marturet., R. S. Mohan, O. Shoham and G. E. Kouba, "The effects of geometry, fluid properties and pressure on the hydrodynamics of gas-liquid cylindrical cyclone separators," Int. J. Multiphas. Flow, vol. 26, 2000, pp. 999-1018.

[2] S. B. Wang, L. Gomez, R. Mohan, O. Shoham, G. Kouba and J. Marrelli, "The State-of-the-Art of Gas-Liquid Cylindrical Cyclone Control Technology: From Laboratory to Field," J. Energ. ResourASME, 2010, pp. 132:1-9.

[3] B.T. Zhao, "A Theoretical Approach to Pressure Drop across Cyclone Separators," Chem. Eng. Technol, vol. 27, 2004, pp. 1105-1108.

[4] B.T. Zhao, "Experimental investigation of flow patterns in cyclones with conventional and symmetrical inlet geometries," Chem. Eng. Technol, vol. 28, 2005, pp. 969-972.

[5] C. J. Tsai, C. N. Liu, S. M. Hung, S. C. Chen, S. N. Uang, Y. S. Cheng, and Y. Zhou, "Novel Active Personal Nanoparticle Sampler for the Exposure Assessment of Nanoparticles in Workplaces," Environ. Sci. Technol, vol. 46, 2012, pp. 4546-4552.

[6] L. Ma, D. Ingham and X. Wen, "Numerical modelling of the fluid and particle penetration through small sampling cyclones," J. Aeroso1. Sci, vol. 31, 2000, pp. 1097-1119

[7] G. Solero, A. Coghe, "Experimental fluid dynamic characterization of a cyclone chamber," Experimental Thermal and Fluid Science, vol. 27,2002 , pp. $87-96$ 
[8] F. Boysan, W. H. Ayers and J. Swithenbank, , "A fundamental mathematical modelling approach to cyclone design," Trans Inst Chem Eng, vol. 60, 1982, pp. 222-230.

[9] T. G. Chuah, J. Gimbun and T.S.Y. Choong, "A CFD study of the effect of cone dimensions on sampling aerocyclones performance and hydrodynamics," Powder Technol, vol. 162, 2006, pp. 126-132.

[10] R. E. DeOtte, "A model for the prediction of the collection efficiency characteristics of a small, cylindrical aerosol sampling cyclone," Aerosol Sci Technol, vol. 12, 1990, pp. 1055-1066.

[11] W. D. Griffiths and F. Boysan, "Computational fluid dynamics(CFD) and empirical modeling of a number of cyclone samplers," J Aerosol Sci, vol. 27, 1996, pp. 281-204.
[12] M. Gautam and A. Streenath, "Performance of a respirable multiinlet cyclone sampler," J Aerosol Sci, vol. 28(7), 1997, pp. 1265-1281.

[13] H. Safikhani, A. B. Akhavan-Behabadi, M. Shams and M. H. Rahimyan, "Numerical simulation of flow field in three types of standard cyclone separators," Adv. Powder Technol, vol. 21, 2010, pp. 435-442.

[14] K. Elsayed and C. Lacor, "The effect of cyclone inlet dimensions on the flow pattern and performance," Appl. Math. Model, vol. 35, 2011, pp. 1952-1968.

[15] Y. F. Qiu, B. Q. Deng and C. N. Kim, "Numerical study of the flow field and separation efficiency of a divergent cyclone," Powder. Technol, vol. 217, 2012, pp. 231-237. 